\title{
Controversies and dilemmas in laryngopharyngeal reflux disease: a new paradigm of airway manifestations of a gastrointestinal disease
}

\author{
P. S. N. Murthy
}

There are quite a few synonyms of the laryngopharyngeal reflux disease (LPRD) such as reflux laryngitis, laryngeal reflux, gastropharyngeal reflux, pharyngosupraesophageal reflux, extraesophageal reflux and atypical reflux, which truly reflects the controversies and dilemmas in LPRD. The etiopathogenesis is uncertain, the symptoms are varied and unexplainable and the diagnosis and treatment is empirical and by trial. Some of these issues will be discussed in this article.

\section{Is laryngopharyngeal reflux a new disease entity?}

The evidence does not seem to suggest this. According to Koufman it is likely that gastroesophageal reflux was recognized in antiquity. In 1618, Fabricius described gastroesophageal (GE) junction referring to it as "cardia". Attributing the term to Galen in 200 AD. Galen coined the term cardia because the symptoms arising from GE junction resemble cardiac symptomatology. But only in 20th century, these two were correlated and the condition gastroesophageal reflux disease (GERD) was coined. Late 19th century saw Chevalier Jackson inventing distal illuminated esophagoscope. He believed that the esophageal strictures were caused by inflammatory disease. Winkelstein in 1935 first described the term "peptic esophagitis" in adults. Cherry and Margulies and Delahunty and Cherry in 1968 in separate articles described GERD affecting the larynx and they attributed the disease to contact ulcers and vocal cord

\section{P. S. N. Murthy}

Department of ENT and Head \& Neck Surgery,

Dr. Pinnamaneni Siddharta Institute of Medical Sciences \&

Research Foundation Chinnaoutapalli,

Gannavaram Mandal, Krishna District

Hyderabad, India

P. S. N. Murthy $(\bowtie)$

e-mail: drmurtypsn@gmail.com grannulaomas. Few case reports appeared in the literature since then attributing a number of symptomatology and disorders to LPRD. GERD patients who do not have heartburn were considered as "atypical GERD" and were thought as vagal mediated reflexes. The condition prevailed till Weiner et al. documented the presence of acid in the pharynx using an esophageal and a pharyngeal $\mathrm{pH}$ probes in 1969 they reported that $78 \%$ had documented LPRD. This analysis also showed that the pattern of reflux in GERD and LPRD is different.

\section{Who should treat LPRD?}

It is interesting to note that the damaging effects of acid reflux into the laryngeal area occurs in a region of the digestive tract or rather upper aerodigestive tract causing a "hands off" between two specialities who individually deal with these patients. The gastroenterologist deals with GERD which occurs below the esophagus and the otolaryngologist deals with LPRD which occurs above the esophagus causing both laryngeal and pharyngeal symptomatology. The message to primary care physicians is that LPRD has to be managed by an otolaryngologist as he is the first consultant to be approached by the patient for essentially symptomatology of pharyngeal and laryngeal disease. The overdiagnosed condition of globus hystericus probably was a unidentified manifestation of LPRD.

\section{How does LPRD and GERD differ?}

Comparably both the entities are separate having different pathophysiological mechanisms, different symptoms, manifestations and response to treatment.

1. Majority of LPRD patients do not have esophagitis or heartburn and only $40 \%$ complain of heartburn and less than $25 \%$ have documented esophagitis. 
2. LPRD patients are predominantly supine, upright and daytime refluxers where as GERD patients are supine and nocturnal refluxers.

3. In GERD there are prolonged periods of exposure of acid wherein LPRD it is only a short exposure of high damage phenomenon.

4. GERD patients have dysmotility and prolonged esophageal acid clearance, which does not happen in LPRD.

5. The primary defect in GERD is lower esophageal dysfunction and in LPRD it is upper esophageal sphincter dysfunction. Having said that, the acid has to traverse the entire esophagus to reach the upper aerodigestive tract. Hence, logistically there should be dysfunction of both the sphincters in LPRD which allows the acid to reach the larynx and pharynx. Probably that explains the coexistence in a percentage of patients.

6. The laryngopharyngeal mucosa is far more susceptible to acid and pepsin exposure than the esophageal mucosa. It is stated that it is 100 times more sensitive to peptic injury than the esophagus and Kaufman in 1991 showed that as few as 3 episodes of reflux per week can result in significant damage to the larynx.

7. The protective barriers in esophagus such as carbonate production, mucosal barrier and peristalisis are absent in the laryngopharynx, making it more susceptible.

\section{What is the symptomatology?}

Here is a pretty long list of head and neck manifestations of LPRD. The symptoms are either intermittent or chronic intermittent and some are proven to be the true symptoms and quite a few symptoms are attributed to LPRD, with or without a valid pathophysiologic basis.

The symptoms can be essentially laryngeal (chronic dysphonias and vocal complaints) or laryngopharyngeal (dysphageas, lump or foreign body sensation in the throat to globus) and pulmonary manifestations. Most of them are studied and there is convincing evidence that the disease can cause these symptomatology. There are some miscellaneous conditions such as SIDS, sinusitis, adenoid hypertrophy, dental caries, aphthous ulcers and OSA have all been attributed to LPRD.

1. Kaufmann in 2000 reported that $50 \%$ of the patients with voice disorders have LPRD by $\mathrm{pH}$ probe.

2. Hanson (1995) showed that $96 \%$ of the 200 patients with chronic laryngitis responded to antireflux treatment.

3. Shaw (1996) also gave similar results of $85 \%$ response in chronic laryngitis.

4. Irwin in 1993 showed that $75 \%$ of the patients with chronic cough did not have any reflux symptoms but have proven and documented evidence of LPRD.
5. Improvement was seen in $73 \%$ of asthmatics in their pulmonary symptomatology with antireflux treatment and improvement in pulmonary function tests with after a three month course of Omeprazole. Asthma can cause large pleural abdominal pressure gradients and anti-asthmatic medications such as Theophylline and beta- 2 antagonists decrease the lower esophageal sphincter pressure resulting in significant reflex.

6. Weiner in 1987 showed that half of his patients with with laryngeal carcinoma had reflux.

7. Kaufmann (1991) presented that $71 \%$ of 31 patients with early glottic carcinomas had reflux.

8. Lewing in 2004 reported a series of 24 hour $\mathrm{pH}$ probes in newly diagnosed cases of laryngeal lesions, which showed that $85 \%$ of them did have LPRD.

Hence the evidence suggests that LPRD do cause symptomatology in pharyngo-laryngo-pulmonary regions. Though most of the evidence is is statistically significant and collloborative, the true relationship of reflux in certain conditions still need confirmation in larger studies.

\section{How do you diagnose LPRD?}

History and clinical symptomatology are are at the top of the list and should be able to point out towards the LPRD as the disease responsible for the symptomatology.

A detailed laryngeal examination by Flexible or 90 degree Hopkin's rod laryngoscopy or even a stroboscopic examination is mandatory to diagnose LPRD. There are six conditions which can be expected to be found - pseudosulcus vocalis, obliteration of the ventricle, hyperemia of vocal folds along with edema, posterior commissure hypertrophy and posterior glottic grannuloma.

Ambulatory 24-hour double probe $\mathrm{pH}$ monitoring is the gold standard of diagnosis with high index of specificity and sensitivity. Two probes, one in the pharynx at the level of the laryngeal inlet and the second probe at 5 $\mathrm{cm}$ above the lower esophageal sphincter. Although it is possible to have some variability, it was found significant day-to-day variation in acid exposure of the proximal eso-phagus but good intraesophageal reproducibility thus demonstrating good specificity but poor sensitivity for LPR monitoring.

Laryngeal sensory testing is not regularly used as a test for LPRD but it appears that the LPRD patients do have laryngeal sensory deficits.

\section{How is LPRD treated?}

There are several treatment options but it needs to be individualized for each individual patient based on their symptoms and the response to treatment. 
1. Behavioural modification: Elevating the head end of the bed by $6-8$ inches, maintaining ideal body weight, not lying down for three hours after eating, avoiding tobacco, foods with fats, spices, acid, alcohol and caffeine and also avoicing drugs that promote larynx, such as calcium channel blockers. Chewing gum can increase salivary bicarbonate production which can neutralize the acid.

2. Pharmacotherapy: Antacids and $\mathrm{H} 2$ receptor blockers need to be taken 3-4 times a day and are effective to neutralize the acid in the stomach. Proton pump inhibitors on a therapeutic trial for at least 6 weeks on a double dose modality is the gold standard treatment of choice. They directly act on parietal cells affecting the key enzyme HK and ATPase in the acid production pathway.

3. Surgical management: Only after the failure of the first two lines of management, opt for Nissan's fundoplication, which aims at tightening the lower esophageal sphincter by rapping a part of the stomach around the lower part of the esophagus. The surgery can be open or endoscopic.

\section{Conclusion}

Laryngopharyngeal reflux disease is becoming more and more commonly diagnosed condition among the pharyn- geal, airway and voice disorders. It is a chronic intermittent disease and dignosis depends upon a detailed history and clinical examination followed by video stroboscopy or flexible endoscopy. LPRD requires double dose long-term PPI therapy than GERD. Most of the times, the disease responds to behavioral modifications and pharmacotherapy and the surgical options are very much restricted to a very few patients.

\section{References}

1. Koufman JA (2002) Laryngopharyngeal reflux 2002. A new paradigm of airway disease. Ear Nose and Throat J Suppl 1: 26

2. Koufman JA, Aviv JE, Casiano RR, Shaw GY (2002) Laryngopharyngeal reflux: Position statement of the committee on speech,voice and swallowing disorders of the American Academy of Otolaryngology and Head and Neck Surgery. Otolaryngol Head Neck Surg 127

3. Koufman J, Satal RT and Toohill R (1996) Laryngopharyngeal reflux: Consensus conference report. 10:215-216

4. Beaver ME, Stasney CR, Weitzel E, Stewart MG, Donovan DT, Parke RB and Rodriguez M. Diagnosis of laryngopharyngeal reflux disease with digital imaging. Otolaryngol Head Neck Surg 128:103-108

5. Scott M Kazuba (2004) Grand Rounds at Baylor School of Medicine www.bcm.edu/oto/grand/11112004.htm dated 11th November 2004 